\title{
Numerical and experimental analysis of the air stream generated by square ceiling diffusers
}

\author{
Marek Jaszczur, ${ }^{*}, 1$, Pawet Madejski ${ }^{1}$, Stawosz Kleszcz ${ }^{1,2}$, Marcin Zych ${ }^{1}$ and Patryk Palej ${ }^{1}$ \\ ${ }^{1}$ AGH University of Technology and Science, 30 Mickiewicza, 30-059 Kraków, Poland \\ ${ }^{2}$ Frapol Sp. z o.o., Kraków, Poland
}

\begin{abstract}
Ceiling diffusers are one of the most important elements of ventilation and air-conditioning installations. They have a significant impact on the speed and temperature distribution of air. The basic task of these devices is to provide the required air stream while maintaining its appropriate parameters in the occupied zone and low noise levels in the room. Square ceiling diffusers are designed for use in low- and medium-pressure ventilation systems. The diffusers allow obtaining 1, 2, 3 or 4-way air supply. Knowledge of the airflow formed by the diffuser is essential in order to ensure the comfort of people staying in a ventilated room. Designers of the diffusers nowadays mainly use advanced software and CFD (Computational Fluid Dynamics) modelling technique. CFD methods allow to quickly analyse the fluid flow. However, an very important problem is the consistency of the results obtained by numerical methods with the results obtained on an experimental way. The work presents a laboratory stand designed to test ceiling diffusers. In this research study the single square ceiling diffuser was experimentally and numerically analysed.
\end{abstract}

\section{INTRODUCTION}

Ventilation of buildings is aimed at ensuring the adequate air quality in rooms. The ventilation system is designed to bring the proper amount of air and ensure the desired air distribution inside the rooms. Improper flow and too small air exchange in the room causes significant discomfort. This can also lead to a sick building syndrome. Living conditions are getting worse. This affects the health of users of rooms as well as the construction of the building. Knowledge and forecasts regarding the flow of indoor air streams are therefore important. They allow to optimise the indoor climate. This translates into energy-saving and environmental protection [1-3]. People in the rooms spend nearly $80 \%$ of their time. Therefore, indoor air quality is an important aspect of the indoor environment.

Ceiling diffusers are one of the most important elements of ventilation and air-conditioning installations. They have a significant impact on the speed and temperature distribution of air. The basic task of these devices is to provide the required air stream while maintaining its appropriate parameters in the occupied zone and low noise levels in the room.

Square diffusers are designed for use in low- and mediumpressure ventilation systems. They are especially recommended for rooms with a height from 3 to 12 meters. The diffusers of this type are widely used in supply and exhaust systems in rooms requiring comfort, such as offices, shopping centres, restaurants, cinemas or so-called "clean rooms" [4, 5].

When selecting this type of diffusers, an important issue is to determine the range zone of the air stream. For this purpose, manufacturers provide appropriate tables, nomograms or figures to determine the extent of the air stream depending on the volume of the airflow. Designers of the diffusers relay at present on CFD modelling technique $[6,7]$. This technique allow to quickly analyse the fluid flow. However, an very important problem is the consistency of the results obtained by numerical methods with the results obtained on an experimental way. For the channel flows DNS [8] and LES [9] provide very accurate results but for the large areas this methods are still beyond practical applications.

It can be noticed, however, that there are significant discrepancies between the data of the different air producers in determining the range of the supply air stream. These discrepancies result mainly from the evaluation methods used by manufacturers. Therefore, it is a valid and up-to-date task to verify the results on an detailed experimental way [10-12].

\section{EXPERIMENTAL SET-UP}

The subject of the research is AL-DV aluminium diffusers equipped with fixed vanes that produce a directed air outflow. The diffusers are designed for mounting on the ceiling. Figure 1 shows the tested diffuser.

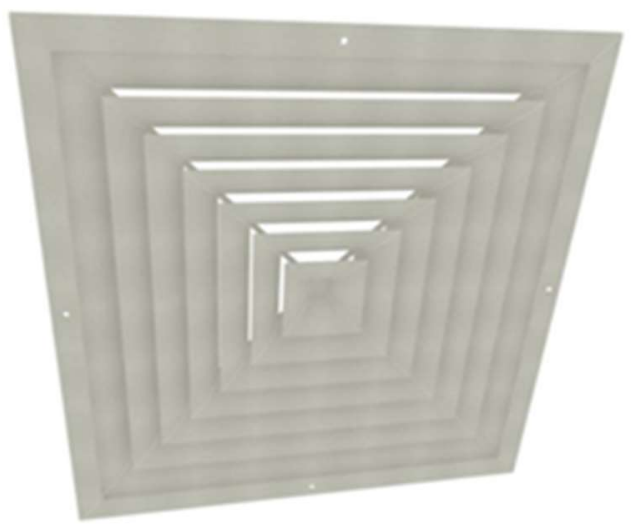

Fig. 1. View on the analysed squire diffuser.

\footnotetext{
*Corresponding author: jaszczur@agh.edu.pl
} 
The front panel of the diffuser is made of aluminium profiles. Diffusers are manufactured in eight standard sizes. Their dimensions (in $\mathrm{mm}$ ) are given in Table 1 according to markings in Figure 2.

Table 1. Dimensions of diffusers in mm (markings as in Fig 2)

\begin{tabular}{|l|l|l|l|}
\hline Size & A & B & C \\
\hline W-1 & 205 & 124 & 245 \\
\hline W-2 & 261 & 180 & 301 \\
\hline W-3 & 317 & 263 & 357 \\
\hline W-4 & 373 & 292 & 413 \\
\hline W-5 & 430 & 349 & 469 \\
\hline W-6 & 459 & 378 & 498 \\
\hline W-7 & 559 & 478 & 598 \\
\hline W-8 & 584 & 503 & 623 \\
\hline
\end{tabular}
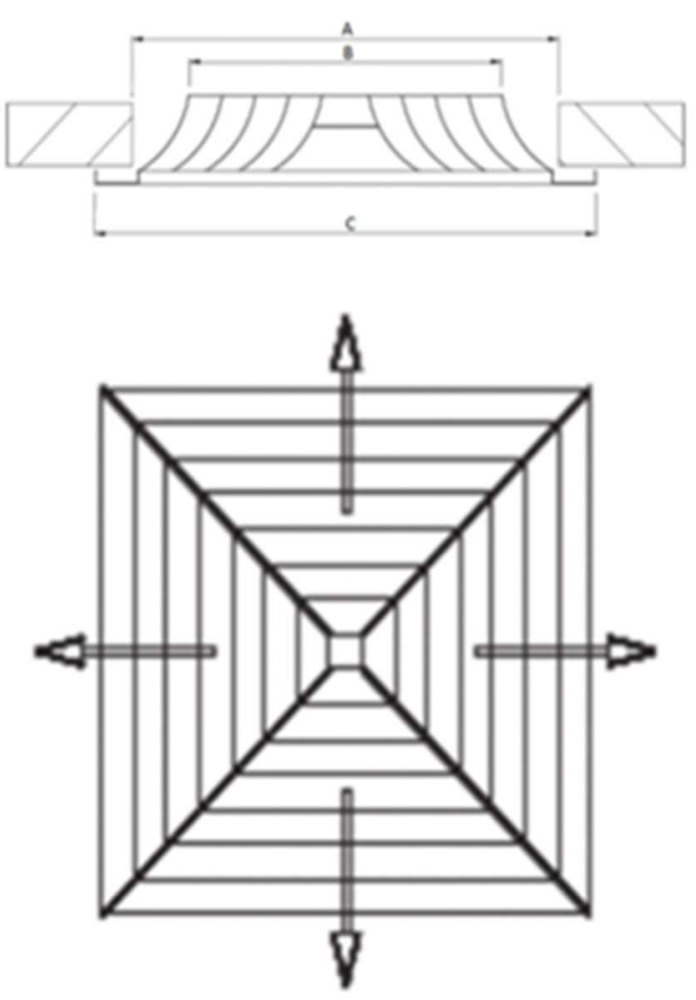

Fig. 2. Dimensions of the squire diffusers and outflow directions of the air stream.

Measurements were made in the grid of measurement points on the $X-Z$ plane as shown in Figure 3. Measurements were made in lines. First, measurements were made on single line for all air streams. Next, the thermoanemometers were lowered to the next measuring line in the Z-axis. The measuring sensors were set at a given height $Z$ at equal intervals of $150 \mathrm{~mm}$ (see Figure 4 ). The measurements were started from the diffuser axis and continue until the measured velocity was lower than $0.5 \mathrm{~m} / \mathrm{s}$. For each the diffusers size tested, the appropriate distances in the vertical axis and different volumetric air flows were selected. The measurement time equal $180 \mathrm{~s}$ and results from every single point were averaged. The spacing of the measurement points was determined on the basis of smoke tests and the size of the supply air stream (Figure 5). As you can see the stream of supply air is symmetrical.

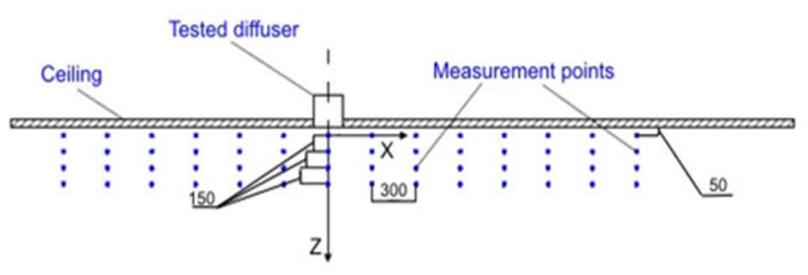

Fig. 3. Measuring points in the $X-Z$ plane.

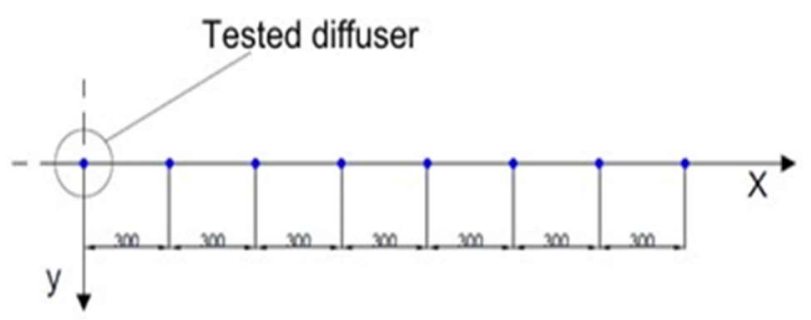

Fig. 4. Measuring points in the $\mathrm{X}-\mathrm{Y}$ plane
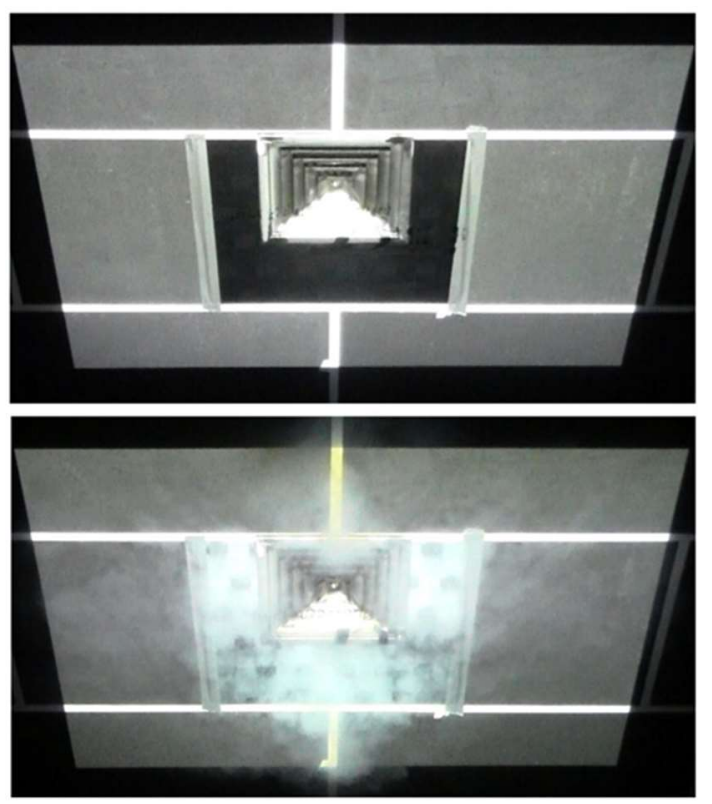

Fig. 5. Photos of the tested diffuser during smoke tests

Measurements were made to determine the range of the stream for the speed of $0.5 \mathrm{~m} / \mathrm{s}$ so-called $\mathrm{L}_{0,5}(\mathrm{~m})$. Figure 6 presents a plot representing the horizontal range $\mathrm{L}_{0.5}$ and the vertical range $\mathrm{H}_{0.5}$ of the stream flowing out of the diffuser. AirDistSys 5000 system was used to measure air velocity and temperature. This system enables multi-point measurements of air velocity and temperature. It is used, inter alia, to assess thermal conditions and the speed distribution in rooms. A measuring system consisting of eight SensoAnemo 5100LSF transducers with an SF3 probe. It allows simultaneous measurement of speed and 
temperature at selected 8 points. The anemometric transducer measures the airflow velocity in the range from 0.05 to $5.00 \mathrm{~m} / \mathrm{s}$. Its uncertainty of measurement is \pm 0.02 $\mathrm{m} / \mathrm{s} \pm 1.5 \%$.

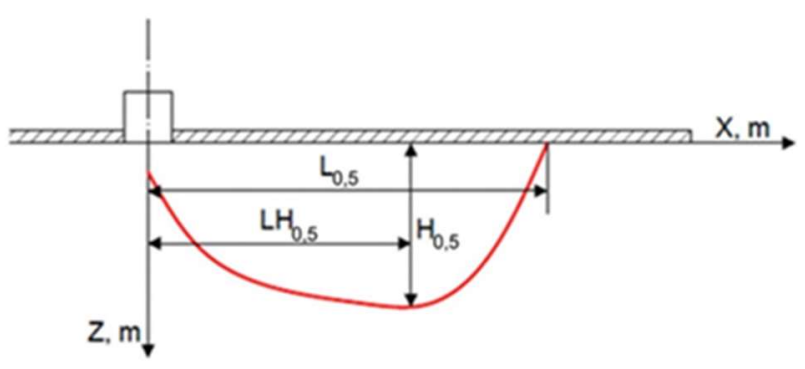

Fig. 6. Horizontal $\mathrm{L}_{0,5}$ and vertical $\mathrm{H}_{0,5}$ range of the stream flowing out of the diffuser

\section{RESULTS}

Figures 7 and 8 show results for the diffuser size $W=3$. The measurements were made for five air flow rates equal to: $100,200,300,400$ and $500 \mathrm{~m} 3 / \mathrm{h}$. Measuring points (thermoanemometers) were placed at distances of $0.3 \mathrm{~m}$ from the diffuser axis. Figure 7 shows the velocity distribution at different distances from the ceiling at the volumetric flow rate of the $400 \mathrm{~m}^{3} / \mathrm{h}$ supplied air stream.

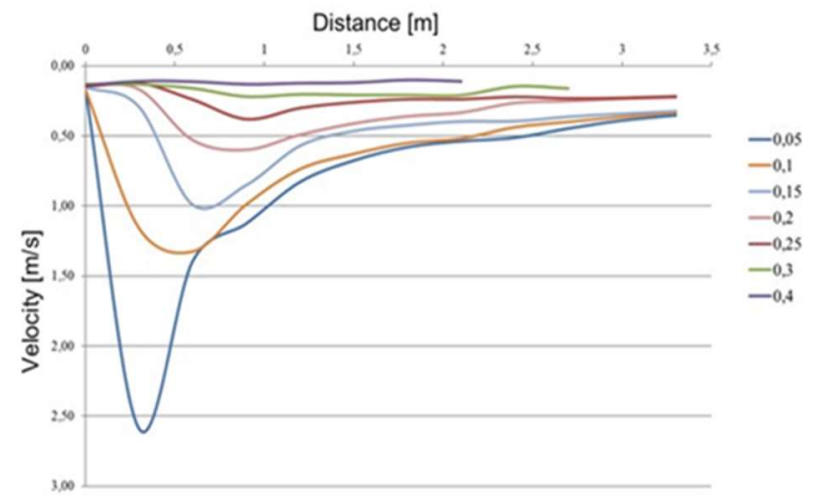

Fig. 7. Distribution of air velocity flowing out of the diffuser $\mathrm{W}-3$ at a volume flow of $400 \mathrm{~m}^{3} / \mathrm{h}$ at different distances from the ceiling (in meters)

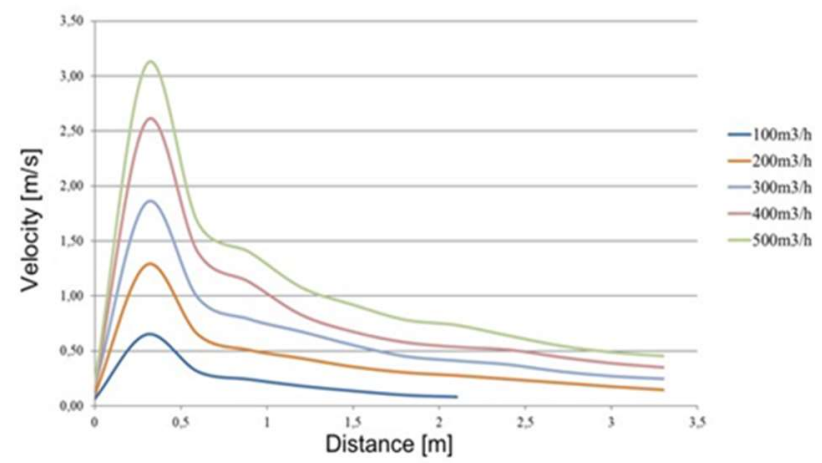

Fig. 8. Distribution of the velocity air blown by the diffuser W3 on the $\mathrm{Z}-\mathrm{X}$ plane $\mathrm{Z}=0.05 \mathrm{~m}$ for different air flow rates
As you can see, the airflow takes place mainly in the $\mathrm{Z}=$ $0.05 \mathrm{~m}$ plane under the ceiling (blue line), in the $\mathrm{Z}-\mathrm{X}$ plane $\mathrm{Z}=0.1 \mathrm{~m}$ (orange line) and $\mathrm{Z}=0.15 \mathrm{~m}$ (bright blue line). In other planes, the air velocity takes on much lower speeds, and the occurring fluctuations in air velocity are insignificant. The velocity distribution in the $Z=0.05 \mathrm{~m}$ plane for different streams is shown in figure 8 .

On the basis of the measurements, the range of the stream was determined, i.e. the distances in which the air velocity reaches $0.5 \mathrm{~m} / \mathrm{s}$ in the X-Z plane, as shown in Figure 9. A graph was obtained with a simplified dependence of the range air stream for the different volumetric flow rate.

The obtained results allowed to determine the range of the stream $\mathrm{L}_{0.5}$ for the entire series of the square diffuser. Figure 10 shows the relationship between the range of the stream $\mathrm{L}_{0.5}$ and the volume flow of supply air.

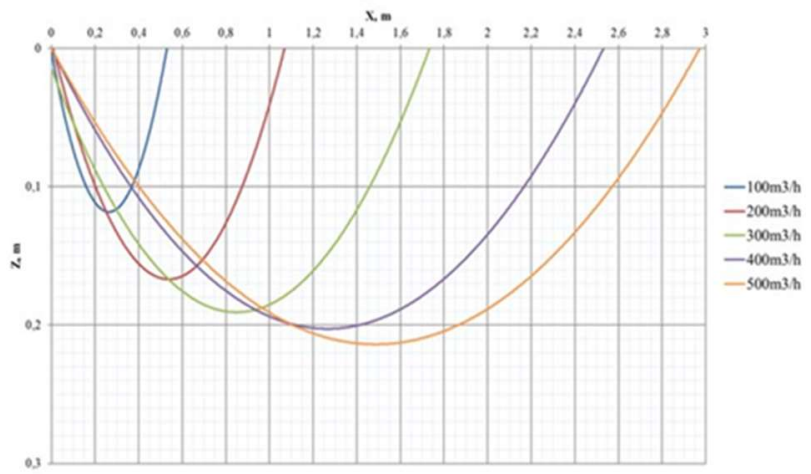

Fig. 9. Range of the air stream for the diffuser W-3 and for the velocity of $0.5 \mathrm{~m} / \mathrm{s}$. Results for various air flow rates

\section{NUMERICAL ANALYSIS}

The numerical simulation of the air stream generated by square ceiling diffuser was conducted using Computational Fluid Dynamics technique and specialised software Star-CCM+ [13]. The 3D geometrical model and numerical mesh consisting of 2896706 polyhedral cells is presented in Figure 11.

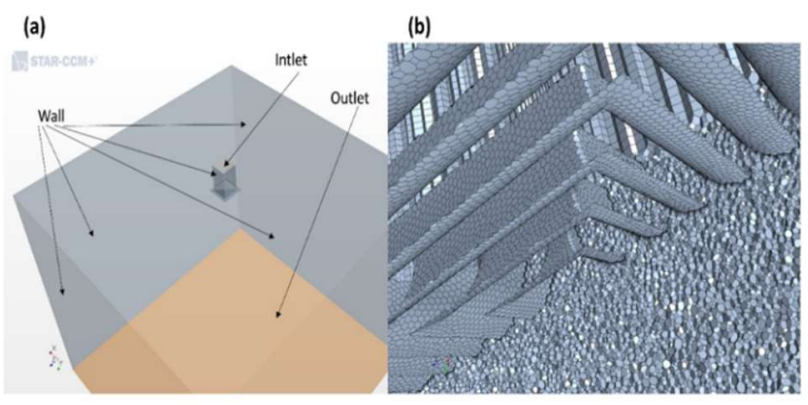

Fig. 11. Geometrical model (a) and numerical mesh in region close to the squire diffuser (b) 
The fluid used in the simulation was the air, treated as incompressible, newtonian gas with a constant density $\left(\rho_{\text {air }}=1.18415 \mathrm{~kg} / \mathrm{m}^{3}\right)$ and dynamic viscosity $\left(\eta_{\text {air }}=1.85508 \mathrm{e}-05 \mathrm{~Pa} \cdot \mathrm{s}\right)$. The turbulent character of fluid flow was simulated using recommended for this type of configurations realizable $\mathrm{k}-\varepsilon$ turbulence model eq.(1)-(3) and the two-layer approach [4].

$$
\begin{gathered}
\partial / \partial t(\rho k)+\nabla \cdot\left(\rho k \mathbf{v}^{-}\right)=\nabla \cdot\left[\left(\mu+\mu_{t} / \sigma_{k}\right) \nabla k\right]+ \\
P_{k}-\rho\left(\varepsilon-\epsilon_{0}\right)+S_{k}
\end{gathered}
$$

$$
\begin{aligned}
\frac{\partial}{\partial t}(\rho \varepsilon)+\nabla & \cdot(\rho \varepsilon \overline{\mathbf{v}})=\nabla \cdot\left[\left(\mu+\frac{\mu_{t}}{\sigma_{\varepsilon}}\right) \nabla \varepsilon\right]+ \\
& +\frac{1}{T_{e}} C_{\varepsilon 1} P_{\varepsilon}-C_{\varepsilon 2} f_{2} \rho\left(\frac{\varepsilon}{T_{e}}-\frac{\epsilon_{0}}{T_{0}}\right)+S_{\epsilon}
\end{aligned}
$$

$$
\mu_{t}=\rho C_{\mu} f_{\mu} k T
$$

where: $\rho$-density $\left(\mathrm{kg} / \mathrm{m}^{3}\right) ; \mathrm{T}$ - turbulent time scale $(\mathrm{s}) ; \mathrm{v}^{-}$ - mean velocity $(\mathrm{m} / \mathrm{s}), \mu-$ dynamic viscosity $(\mathrm{Pa} \cdot \mathrm{s}), \mathrm{C}_{\mu}$, $\sigma_{\mathrm{k}}, \sigma_{\varepsilon}, \mathrm{C}_{\varepsilon 1}, \mathrm{C}_{\varepsilon 2}-$ model coefficients, $\mathrm{f}_{\mu}, \mathrm{f}_{2}-$ damping functions, $\mathrm{P}_{\mathrm{k}}, \mathrm{P}_{\varepsilon}-$ production terms, $\mathrm{S}_{\mathrm{k}}, \mathrm{S}_{\epsilon}-$ userspecified source terms. The velocity distribution and velocity vectors in the vertical plane of the analysed region is presented in Figure 12 and 13.

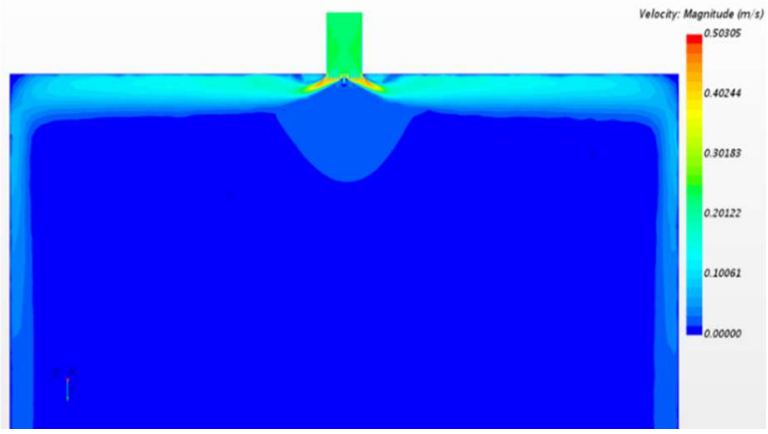

Fig. 12. Velocity distribution of air flowing through squire ceiling diffuser W-5 at volumetric flow of $400 \mathrm{~m} 3 / \mathrm{h}$

The results in the form of air velocity distribution at a volume flow rate of $400 \mathrm{~m} 3 / \mathrm{h}$ and for diffuser size $\mathrm{W}-5$ at different distances from the ceiling are presented in Figure 14. The velocity profiles (Fig. 14) have the same shape as results obtained from the experimental measurement. The maximum value of velocity is dependent on analysed variant and size of diffuser. For the same volumetric flowrate, the maximum velocity value is equal to 2.5 and $0.4 \mathrm{~m} / \mathrm{s}$ for W-3 and W-5 respectively. Increase of diffuser dimensions reduces the velocity of air flows out from diffuser.

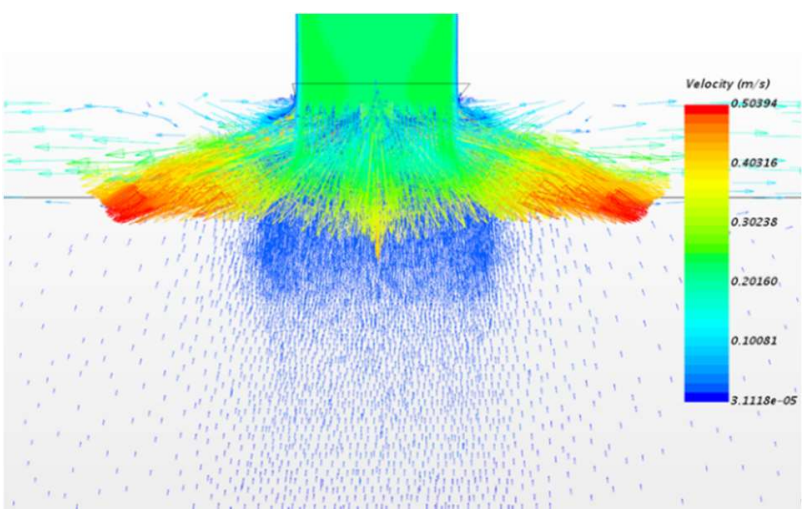

Fig. 13. Velocity vectors of air flowing through squire ceiling diffuser W-5 at volumetric flow of $400 \mathrm{~m} 3 / \mathrm{h}$

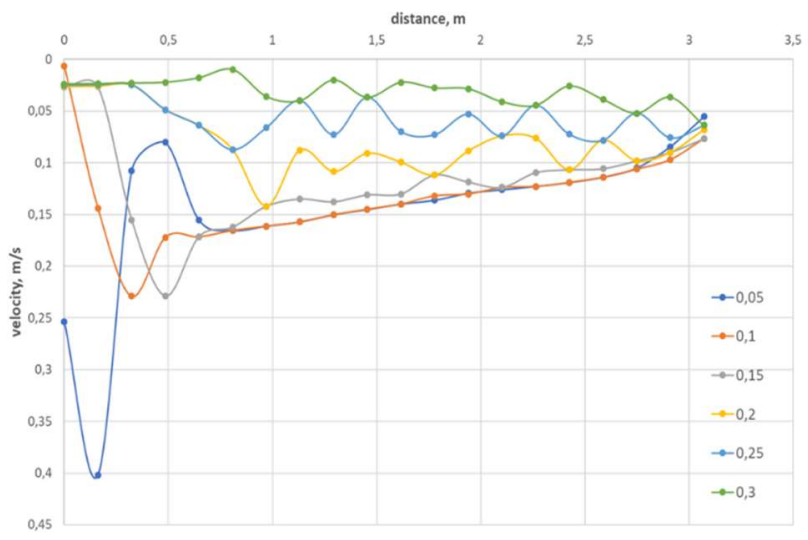

Fig. 14. Air velocity distribution from numerical simulation, flowing out of the diffuser W-5 at a volume flow of $400 \mathrm{~m} 3 / \mathrm{h}$ and at different distances from the ceiling (in meters)

\section{CONCLUSIONS}

One of the most important tasks of ventilation systems is the proper organisation of air separation and the control against harmful emissions in the facility. Typically, airflow and the spread of pollutants take place in a variety of ways. In order to meet general requirements, the airflow can be shaped in various ways, obtaining the desired effects by means of various ventilation elements. The diffusers shape the initial velocity distribution of the stream. By using the appropriate shape of the supply element, the stream can be focused or dispersed and its range can be changed. Knowledge about the air streams shaped by diffusers is therefore important in the design process of ventilation systems.

ACKNOWLEDGMENTS. The present work was partially supported by the Polish Ministry of Science (Grant AGH No. 16.16.210.476).

\section{References}

1. H. Awbi, Ventilation of buildings. London: Taylor and Francis (2003) 
2. A. Pełech Wentylacja i klimatyzacja. Podstawy. Wrocław: Oficyna Wydawnicza Politechniki Wrocławskiej. (in polish) (2013)

3. M. Jaszczur, M. Borowski, D. Satola, S. Kleszcz, M. Karch Analysis of the temperature, humidity and total efficiency of the air handling unit with a periodic counterflow heat exchanger, Thermal Science, 23 (2019)

4. M.A. Aziz, A.M. Gad Ibrahim Experimental and numerical study of influence of air ceiling diffusers on room air flow characteristics, Energy and Buildings, 55 (2012)

5. R.H. Mohammed A simplified method for modeling of round and square ceiling diffusers,. Energy and Buildings, 64 (2013)

6. J. Szmyd, M.Branny, M. Karch, W. Wodziak, M. Jaszczur, R. Nowak Experimental and numerical analysis of the air flow in T-shape channel flow, Archives of Mining Sciences, 58 (2013)

7. P.V. Nielsen Fifty years of CFD for room air distribution, Build. Environ. 91 (2015)

8. M. Jaszczur, M. Portela Numerical data for reliability of LES for non-isothermal multiphase turbulent channel flow, ERCOFTAC 12 (2008)

9. M. Jaszczur, Numerical analysis of a fully developed non-isothermal particle-laden turbulent channel flow, Archives of Mechanics, 8 (2011)

10. M. Borowski, M. Jaszczur, D. Satoła, M. Karch Air flow characteristics of a room with air vortex diffuser, MATEC Web of Conferences, 240 (2018)

11. M. Jaszczur, M. Borowski, M. Karch, M. Branny The study of the velocity field of the air flowing the swirl diffusers using PIV method. EPJ Web of Conferences, 143 (2018)

12. C.S. From, E. Sauret, S.W. Armfield, S.C. Saha, Y.T $\mathrm{Gu}$, Turbulent dense gas flow characteristics in swirling conical diffuser, Computer Fluids, 149 (2017)

13. Simcenter STAR-CCM+® Version 13.06 Siemens PLM Software (2018) 